\title{
A Case of Moderate Sprue-Like Enteropathy Associated With Telmisartan
}

\author{
Aurelio Negro ${ }^{\mathrm{a}}$, Loredana De Marco ${ }^{\mathrm{b}}$, Valentina Cesario ${ }^{\mathrm{a}, \mathrm{c}}$, Rosaria Santia, \\ Maria Chiara Boni ${ }^{\mathrm{a}}$, Magda Zanelli ${ }^{\mathrm{b}}$
}

\begin{abstract}
Sprue-like enteropathy associated with olmesartan medoxomil use has been recently reported. Its clinical manifestations include diarrhea, weight loss and malabsorption. Duodenal biopsies show villous atrophy (VA) with or without intraepithelial lymphocytosis and inflammation of the lamina propria. Serology for celiac disease (CD) is negative and gluten-free diet does not result in clinical improvement. Symptoms resolve after olmesartan discontinuation. Followup biopsies show recovery/improvement of the duodenum. Whether sprue-like enteropathy is a specific adverse reaction to olmesartan or rather a class effect of angiotensin-receptor blockers (ARBs) remains a controversial issue. We report a case of sprue-like enteropathy associated with telmisartan. A 52-year-old man presented with chronic diarrhea, abdominal discomfort and significant weight loss. In the last 3 years, he had been treated with telmisartan $40 \mathrm{mg} /$ day for hypertension after right adrenalectomy for an aldosterone-producing adenoma. Laboratory investigations showed no significant abnormalities: $\mathrm{Hb} 13.6 \mathrm{~g} / \mathrm{dL}$, serum albumin $3.9 \mathrm{~g} / \mathrm{dL}$, total cholesterol $193 \mathrm{mg} / \mathrm{dL}$, serum creatinine $0.99 \mathrm{mg} / \mathrm{dL}$, sodium $143.6 \mathrm{mmol} / \mathrm{L}, \mathrm{K}^{+} 4.3 \mathrm{mmol} / \mathrm{L}$, calcium $9.3 \mathrm{mg} / \mathrm{dL}$, phosphorus $3.9 \mathrm{mg} / \mathrm{dL}$ and 25-OH-D3 $27.7 \mathrm{ng} /$ $\mathrm{mL}$. Duodenal histology showed subtotal VA and inflammation of the lamina propria. CD serology was negative. HLA-DQ typing showed absence of the DQ2/DQ8 haplotypes. After telmisartan discontinuation, patient's symptoms subsided, and his body weight increased despite persistence of a gluten-containing diet. Follow-up biopsies at 3 showed progressive duodenal recovery. Very few cases of spruelike enteropathy associated with ARBs other than olmesartan have been reported. Our case of telmisartan-associated enteropathy further suggests that sprue-like disease may be a class effect of ARBs.
\end{abstract}

Keywords: Sprue-like enteropathy; Telmisartan

Manuscript submitted April 21, 2017, accepted May 2, 2017

${ }^{a}$ Internal Medicine, Specialized Center of Secondary Hypertension and Gastroenterology, IRCCS-Arcispedale Santa Maria Nuova, Reggio Emilia, Italy bUnit of Pathology, Department of Oncology and Advanced Technologies, IRCCS-Arcispedale Santa Maria Nuova, Reggio Emilia, Italy

${ }^{\mathrm{c} C}$ Corresponding Author: Valentina Cesario, Internal Medicine, Specialized Center of Secondary Hypertension and Gastroenterology, IRCCS-Arcispedale Santa Maria Nuova, Reggio Emilia, Italy.

Email: cesario.valentina@gmail.com

doi: https://doi.org/10.14740/jocmr3047w

\section{Introduction}

Drug-associated small bowel enteropathy mimicking celiac disease (CD) has been previously reported in association with azathioprine, mycophenolate mofetil, methotrexate, and nonsteroidal anti-inflammatory drugs [1-3]. Recently, a sprue-like enteropathy associated with the use of olmesartan, an angiotensin II type 1 receptor blocker (ARB), has been described in two case series $[4,5]$. The main clinical manifestations, which develop several months or years after the initiation of olmesartan therapy, include chronic diarrhea, steatorrhea, weight loss and malabsorption. Anemia, hypoalbuminemia and electrolyte abnormalities are present in many patients. Symptoms may be very severe and sometimes life-threatening. Duodenal biopsies show variable degrees of villous atrophy (VA), with or without intraepithelial lymphocytosis or subepithelial collagen deposition. Crypt hypertrophy and inflammation of the lamina propria may also be found. In addition to small bowel involvement, lymphocytic gastritis and/or microscopic colitis may be present as well [4]. Serology for CD is negative and gluten-free diet is not beneficial. However, a high proportion of patients with olmesartan-associated enteropathy carry the HLA-DQ2 and/or DQ8 haplotypes, which are also present in most patients with CD [4]. Discontinuation of olmesartan results in clinical and histologic improvement, while inadvertent rechallenge leads to prompt recurrence of symptoms $[4,5]$.

Based on the published case series and the reports received by FDA's Adverse Event Reporting System, FDA included sprue-like enteropathy among the adverse reactions to olmesartan in July 2013. Since then, other case series and single case reports have further supported the association between olmesartan and sprue-like enteropathy [6-12]. Whether this association is limited to olmesartan or concerns the whole ARBs class is an unresolved issue. A large cohort study has shown that only olmesartan, unlike other ARBs, is associated with an increased risk of severe intestinal malabsorption resulting in hospitalization [13]. On the other hand, single cases of sprue-like enteropathy associated with other ARBs (irbesartan, valsartan, and losartan, respectively) have been reported $[9,14,15]$. At our knowledge, a case of terminal ileitis and another of sprue-like enteropathy associated with telmisartan have been observed $[16,17]$. Herein, we report a case of an only moderate telmisartan-induced sprue-like enteropathy. This case further corroborates this condition as a class effect 


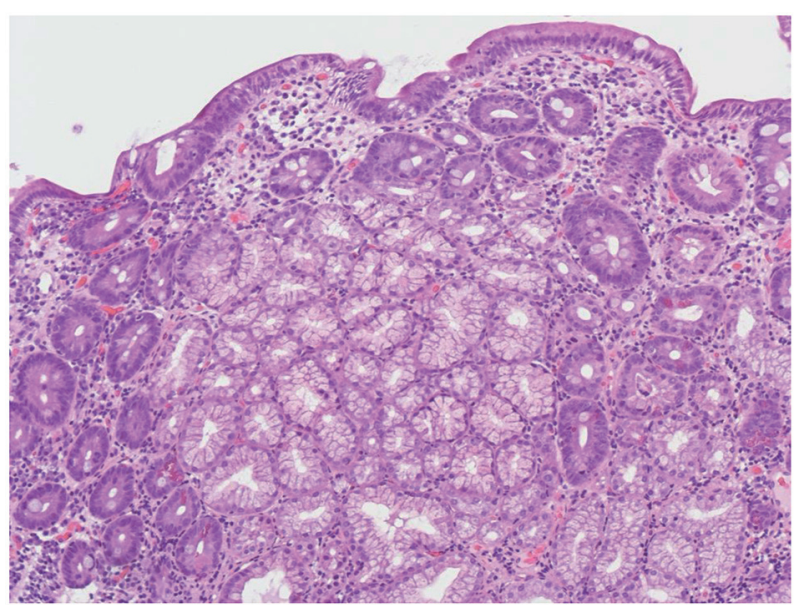

Figure 1. Higher-power view of duodenal mucosal, before suspension of telmisartan (H\&E, original magnification $\times 200)$. The biopsy shows partial villous atrophy and mixed inflammatory infiltration of the lamina propria.

of ARBs.

\section{Case Report}

A 52-year-old man presented to our hospital in December 2016 for chronic diarrhea and a $7 \mathrm{~kg}$ weight loss over the preceding 6 months. He reported 4 - 5 daily evacuations of voluminous, pale, malodorous, non-bloody diarrhea associated with bloating. He also complained of fatigue, nausea, abdominal discomfort, and loss of appetite with an usual gluten-containing diet. In the last 3 years, he had been taking telmisartan $40 \mathrm{mg} /$ day for mild hypertension. The patient was not taking any other medication. His medical history was remarkable for right adrenal gland removal for an aldosterone-secreting cortical adenoma 3 years before. On admission, his blood pressure was 128/81 mm Hg and his body weight was $69 \mathrm{~kg}$. Physical examination showed mild abdominal tenderness. Laboratory tests revealed no significant abnormalities: $\mathrm{Hb} 13.6 \mathrm{~g} / \mathrm{dL}$, serum albumin $3.9 \mathrm{~g} / \mathrm{dL}$, total cholesterol $193 \mathrm{mg} / \mathrm{dL}$, serum creatinine $0.99 \mathrm{mg} / \mathrm{dL}$, sodium $143.6 \mathrm{mmol} / \mathrm{L}, \mathrm{K}^{+} 4.3 \mathrm{mmol} / \mathrm{L}$, calcium $9.3 \mathrm{mg} / \mathrm{dL}$, phosphorus $3.9 \mathrm{mg} / \mathrm{dL}$ and 25-OH-D3 (27.7 $\mathrm{ng} / \mathrm{mL}$ ). Fecal occult blood tests repeatedly yielded negative results, as well as the stool cultures for Salmonella, Shigella, Campylobacter and Enteropathogenic Escherichia coli and Clostridium difficile antigen and A/B toxins assays. Repeated microscopic stool evaluation showed neither ova nor Cryptosporidium nor other Coccidii. Giardia and Entamoeba histol$y$ tica/dispar antigens detection tests were negative. Anti-tissue transglutaminase antibodies were absent. HLA-DQ typing was negative for $\mathrm{CD}$ permissive haplotypes (HLA DQ2/8). Immunofluorescence assay for antinuclear antibodies was negative. Both the ultrasound and thoracic/abdominal computed tomography scan showed normal findings. The patient underwent an esophagogastroduodenoscopy (EGDS) and biopsies of the duodenum revealed a mild and partial VA with superficial erosions and without increase in intraepithelial lymphocytes (Fig.

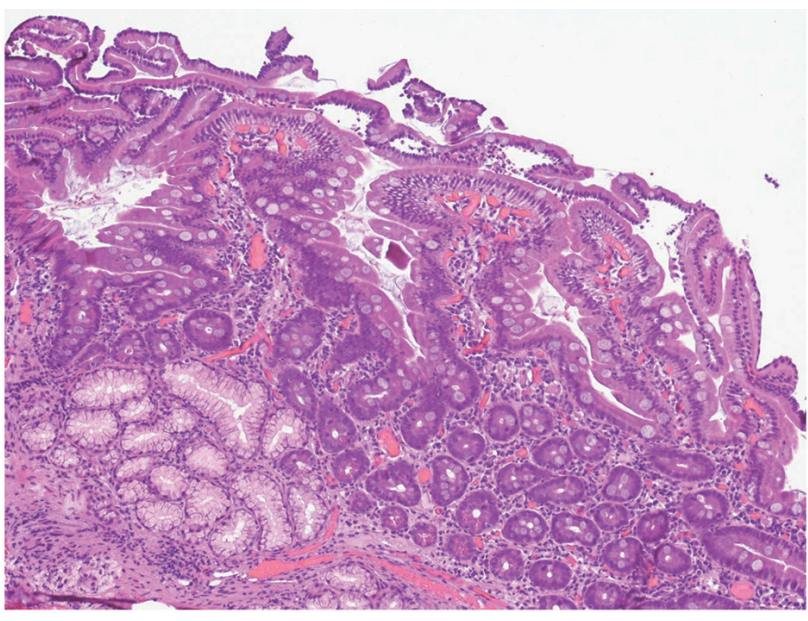

Figure 2. Higher-power view of duodenal mucosal, after suspension of telmisartan $(H \& E$, original magnification $\times 200)$. The biopsy shows initial villous regeneration 3 months after telmisartan withdrawal. The vills are irregular and serrated.

1). A colonoscopy was performed and histological evaluation of multiple colonic biopsies showed a moderate inflammatory infiltrate with a mild increase of eosinophils in the left colon.

In the suspect of a drug-related intestinal damage, telmisartan was withdrawn. Suspension of telmisartan relieved all the symptoms within 1 week about. He was then discharged with definitive suspension of telmisartan. His blood pressure has been normal since then, hence the anti-hypertensive drug has not been replaced. Three months after drug withdrawal, an EGD of follow-up was performed and histology of duodenal biopsies showed a complete restitution of villi architecture (Fig. 2). The patient regained 6-kg body weight in the next 5 months after discharge.

\section{Discussion}

The clinicopathologic features of our patient closely resemble those observed in the case series of olmesartan-associated enteropathy reported by Rubio-Tapia et al [4]. Indeed, patient's symptoms at presentation were chronic diarrhea and substantial weight loss but no features of malabsorption, which had developed 3 years after the initiation of telmisartan therapy. Laboratory examinations showed negative CD serology. Finally, our patient had clinical and histologic recovery after drug discontinuation. Though we did not test the response to a gluten-free diet, we excluded seronegative $\mathrm{CD}$ based on both the absence of HLA-DQ2/DQ8 haplotypes and the clinicopathologic response to ARB suspension despite maintenance of a gluten-containing diet.

Our case of telmisartan-associated enteropathy adds to a few cases associated with ARBs other than olmesartan. It further suggests that sprue-like enteropathy may be a class effect of ARBs, though the risk of this severe adverse reaction is higher with olmesartan compared to other ARBs [9, $14,15]$. Despite a growing body of evidence supporting the association between ARBs and sprue-like enteropathy, we 
acknowledge that a clear-cut demonstration of a cause-andeffect relationship is lacking at present. Moreover, ARB-associated sprue-like enteropathy is nowadays believed to be a rare event, given the enormous number of patients treated with ARBs who do not develop intestinal symptoms, both in clinical trials and in the common clinical practice. However, in a recent French observational study of angiotensin-converting enzyme inhibitors (ACEIs) and ARBs users, olmesartan was associated with an adjusted rate ratio of 2.49 for hospitalization with a discharge diagnosis of intestinal malasorbption compared to ACEI and a rate ratio of 3.17 compared with other ARBs. Notably, the incidence of hospitalization for olmesartan was also associated to treatment duration with a rate ratio of 0.76 for treatment period less than 1 year, 4.36 for treatment from 1 to 2 years, and 10.2 for treatment period greater than 2 years. Therefore, this time of exposure-related increased incidence of hospitalization suggests that olmesartan-induced enteropathy may develop slowly [18]. However, as in our case, the ARBs can induce more subtle intestinal damage and then milder sprue-like enteropathy. Whether this milder presentation represents a stage in the ultimate development of severe sprue-like enteropathy or a limited injury remains to be determined [19]. Therefore, it is conceivable that clinical spectrum of ARBs-related enteropathy may be varying from mild to severe and life-threatening disease, with only the bulk of the iceberg observed until now.

The mechanisms underlying ARB-associated enteropathy are still unknown. The long delay between the onset of ARB therapy and the development of symptoms suggests a cell-mediated immunologic damage [4]. ARB-mediated inhibition of transforming growth factor $\beta$ (TGF- $\beta$ ) has been suggested as a possible mechanism, given the crucial role of TGF- $\beta$ in the gut immune homeostasis [4]. Furthermore, an increased number of $\mathrm{CD}^{+}$cells have been observed in the duodenal biopsies of patients with olmesartan-related enteropathy [16]. This finding suggests that either a local expansion of cytotoxic $\mathrm{CD}^{+} \mathrm{T}$ cells or an abnormal intestinal regulation of $\mathrm{T}$ cell activity may play a role in ARB-associated enteropathy. Alternatively, apoptosis of enterocytes resulting from increased binding of angiotensin II to subtype 2 receptor (AT2) has been hypothesized [12]. Indeed, angiotensin II may promote enterocyte apoptosis through binding to AT2, which is expressed in the duodenum and jejunum $[20,21]$. ARB-induced subtype 1 receptor (AT1) blocking leads to increased circulating levels of angiotensin II, which could result in AT2 overstimulation, increased apoptosis of enterocytes and eventually VA.

If the mechanism underlying ARB-related enteropathy is elusive, even more so is the reason accounting for the differences in the frequency of this adverse reaction among the various ARBs.

\section{Conclusion}

Prior to this report, telmisartan has been involved in only two reports of ARB-associated sprue-like enteropathy. Our case further suggests that this rare adverse effect might be a class effect of ARBs rather than a single drug. However, rare it might be, this adverse reaction is severe and potentially life-threaten- ing. We believe that in the setting of a sprue-like enteropathy with negative $\mathrm{CD}$ serology in a patient on $\mathrm{ARB}$ medication, drug discontinuation must be considered.

\section{Financial Support}

All authors disclosed no financial support.

\section{Abbreviations}

AT1: angiotensin II subtype 1 receptor; AT2: angiotensin II subtype 2 receptor; ARB: angiotensin-receptor blocker; CD: celiac disease; EGD: esophagogastroduodenoscopy; TGF- $\beta$ : transforming growth factor $\beta$; VA: villous atrophy

\section{References}

1. Ziegler TR, Fernandez-Estivariz C, Gu LH, Fried MW, Leader LM. Severe villus atrophy and chronic malabsorption induced by azathioprine. Gastroenterology. 2003;124(7):1950-1957.

2. Kamar N, Faure P, Dupuis E, Cointault O, Joseph-Hein $\mathrm{K}$, Durand D, Moreau J, et al. Villous atrophy induced by mycophenolate mofetil in renal-transplant patients. Transpl Int. 2004;17(8):463-467.

3. Bosca MM, Anon R, Mayordomo E, Villagrasa R, Balza $\mathrm{N}$, Amoros C, Corts JR, et al. Methotrexate induced spruelike syndrome. World J Gastroenterol. 2008;14(45):70097011.

4. Rubio-Tapia A, Herman ML, Ludvigsson JF, Kelly DG, Mangan TF, Wu TT, Murray JA. Severe spruelike enteropathy associated with olmesartan. Mayo Clin Proc. 2012;87(8):732-738.

5. DeGaetani M, Tennyson CA, Lebwohl B, Lewis SK, Abu Daya H, Arguelles-Grande C, Bhagat G, et al. Villous atrophy and negative celiac serology: a diagnostic and therapeutic dilemma. Am J Gastroenterol. 2013;108(5):647653.

6. Dreifuss SE, Tomizawa Y, Farber NJ, Davison JM, Sohnen AE. Spruelike enteropathy associated with olmesartan: an unusual case of severe diarrhea. Case Rep Gastrointest Med. 2013;2013:618071.

7. Stanich PP, Yearsley M, Meyer MM. Olmesartan-associated sprue-like enteropathy. J Clin Gastroenterol. 2013;47(10):894-895.

8. Nielsen JA, Steephen A, Lewin M. Angiotensin-II inhibitor (olmesartan)-induced collagenous sprue with resolution following discontinuation of drug. World J Gastroenterol. 2013;19(40):6928-6930.

9. Marthey L, Cadiot G, Seksik P, Pouderoux P, Lacroute J, Skinazi F, Mesnard B, et al. Olmesartan-associated enteropathy: results of a national survey. Aliment Pharmacol Ther. 2014;40(9):1103-1109.

10. Khan AS, Peter S, Wilcox CM. Olmesartan-induced enteropathy resembling celiac disease. Endoscopy. 
2014;46(Suppl 1 UCTN):E97-98.

11. Theophile H, David XR, Miremont-Salame G, Haramburu F. Five cases of sprue-like enteropathy in patients treated by olmesartan. Dig Liver Dis. 2014;46(5):465469.

12. Ianiro $\mathrm{G}$, Bibbo S, Montalto M, Ricci R, Gasbarrini A, Cammarota G. Systematic review: Sprue-like enteropathy associated with olmesartan. Aliment Pharmacol Ther. 2014;40(1):16-23.

13. Basson M, Mezzarobba M, Weill A, et al. Severe malabsorption associated with olmesartan: a French nationwide cohort study. Gastroenerology. 2014;146(S1):S114.

14. Herman ML, Rubio-Tapia A, Wu TT, Murray JA. A case of severe sprue-like enteropathy associated with valsartan. ACG Case Rep J. 2015;2(2):92-94.

15. Negro A, Rossi GM, Santi R, Iori V, De Marco L. A case of severe sprue-like enteropathy associated with losartan. J Clin Gastroenterol. 2015;49(9):794.

16. Cyrany J, Vasatko T, Machac J, Nova M, Szanyi J, Kopacova M. Letter: telmisartan-associated enteropathy - is there any class effect? Aliment Pharmacol Ther. 2014;40(5):569-570.
17. Mandavdhare HS, Sharma V, Prasad KK, Kumar A, Rathi M, Rana SS. Telmisartan-induced sprue-like enteropathy: a case report and a review of patients using non-olmesartan angiotensin receptor blockers. Intest Res. 2017;15(3):419-421.

18. Basson M, Mezzarobba M, Weill A, Ricordeau P, Allemand H, Alla F, Carbonnel F. Severe intestinal malabsorption associated with olmesartan: a French nationwide observational cohort study. Gut. 2016;65(10):1664-1669.

19. Lagana SM, Braunstein ED, Arguelles-Grande C, Bhagat G, Green PH, Lebwohl B. Sprue-like histology in patients with abdominal pain taking olmesartan compared with other angiotensin receptor blockers. J Clin Pathol. 2015;68(1):29-32.

20. Rubio-Tapia A. Sprue-like enteropathy associated with olmesartan - broadening the differential diagnosis of enteropathy. Aliment Pharmacol Ther. 2014;40(1112):1362-1363.

21. Sun L, Wang W, Xiao W, Liang H, Yang Y, Yang H. Angiotensin II induces apoptosis in intestinal epithelial cells through the AT2 receptor, GATA- 6 and the Bax pathway. Biochem Biophys Res Commun. 2012;424(4):663-668. 